\title{
Prevalência de helmintos parasitos dos peixes do açude Pereira de Miranda e dos viveiros do DNOCS (Pentecoste, Ceará, Brasil)
}

\section{Evalution of parasitism by helminths in the fishes from the dam "Pereira de Miranda" and vivarium from "DNOCS" (Pentecoste, Ceará, Brazil)}

\author{
Anna Kohn, ${ }^{* 1}$ Berenice M.M. Fernandes, ${ }^{*}$ Maria de Fatima D. Baptista-Farias, ${ }^{*}$ Simone C. Cohen, ${ }^{*}$ Antonia Lucia dos \\ Santos, ${ }^{*}$ Maria Clara Pamplona-Basilio, ${ }^{*}$ Marcelo J. A. F. Vieira, ${ }^{* *}$ Vicente A. Feitosa ${ }^{\star *}$
}

\begin{abstract}
Resumo
Foi realizada uma avaliação da helmintofauna dos peixes do Departamento Nacional de Obras Contra as Secas, DNOCS, Pentecoste, Ceará, Brasil. Foram examinados 568 peixes pertencentes a 27 diferentes espécies em abril de 1996, agosto de 1998, novembro de 2000 e abril de 2002, coletados no Açude Pereira de Miranda e nos vivieiros do Centro de Pesquisas Rodolfo Von Ihering. Do total de peixes examinados, $164(28,87 \%)$ encontravam-se parasitados por helmintos, incluindo Monogenea, Cestoda, Digenea e Nematoda, isolados ou em associações. Monogenea apresentou o mais alto índice de prevalência de parasitismo $(20,7 \%)$.
\end{abstract}

Palavras-chave: peixe de água doce, parasitos, helmintos, Brasil.

\begin{abstract}
A helminthological evaluation was made on fishes of the "Departamento de Obras Contra as Secas-DNOCS", Pentecoste, Ceará, Brazil. Five hundred sixty eight fishes belonging to 27 different species were examined on April 1996, August 1998, November 2000 and April 2002, collected in the dam "Pereira de Miranda" and in viviparus of the research center. Of the total of fishes examined, 164 (28,87\%) were parasitized by helminths, including Monogenea, Cestoda, Digenea and Nematoda isolated or in associations. Monogenea presented the higher prevalence of parasitism (20,7\%) probably due to diret life cicle of these parasites. With tables and figures.
\end{abstract}

Keywords: freshwater fishes, parasites, helminths, Brazil.

\section{Introdução}

Atualmente, poucos trabalhos têm sido realizados enfocando avaliações da composição da fauna helmintológica de peixes de água doce no país (Kohn et al., 1987; Kohn, 1988; Pavanelli et al., 1997; Machado et al., 2000). Segundo Pavanelli et al. (1997), a maioria dos estudos enfatiza principalmente aspectos taxonômicos de uma espécie ou grupo de parasitas.

Em função da necessidade de aumentar a produtividade da pesca nos açudes da região Nordeste do Brasil, o DNOCS aclimatou e introduziu em seus reservatórios espécies oriundas de outras regiões do país, como o tucunaré (Cichla ocellaris) do rio Amazonas, a pescada (Plagioscion squamosissimus) do rio Parnaíba e a exótica tilápia do Nilo (Oreochromis niloticus) procedente do continente africano.

Avaliações parasitológicas dos peixes de diversas estações de piscicultura do Departamento Nacional de Obras Contra as Secas, DNOCS, foram realizadas em 1992 por Békési. Com a finalidade de ampliar e atualizar o conhecimento do parasitismo por helmintos de peixes represados, foram realizados estudos no Açude Pereira de Miranda e nos viveiros do Centro de Pesquisas Rodolfo Von Ihering.

\section{Material e métodos}

Foram realizadas quatro excursões ao Centro de Pesquisas em Aqüicultura Rodolfo Von Ihering, para coleta de helmintos parasitos dos peixes dos viveiros e do açude Pereira de Miranda, nos meses de abril de 1996, agosto de 1998, novembro de 2000 e abril de 2002, tendo sido examinados 568 peixes pertencentes a 27 diferentes espécies. Do açude Pereira de Miranda foram examinadas 18 espécies diferentes de peixes num total de 297 exemplares e dos viveiros foram examinados 271 peixes pertencentes a 23 diferentes espécies.

Os peixes do açude foram coletados com redes de espera, tarrafas e anzol e os dos viveiros com redes de arrasto e puçás e transportados para o laboratório onde foram conservados vivos em galões, para serem examinados. Os peixes foram medidos e pesados antes de serem examinados e os helmintos encontrados foram processados de acordo com a metodologia específica para cada grupo (Kohn et al., 1985) e identificados no Laboratório de Helmintos Parasitos de Peixes, Instituto Oswaldo Oswaldo Cruz, FIOCRUZ, Rio de Janeiro. Os termos ecológicos são citados de acordo com Bush et al., 1997 e os nomes científicos das espécies de peixes foram fornecidos pelos pesquisadores do DNOCS.

\footnotetext{
* Laboratório de Helmintos Parasitos de Peixes, Departamento de Helmintologia, Instituto Oswaldo Cruz, Av. Brasil 4365, Rio de Janeiro, RJ, Brasil. E-mail: annakohn@ioc.fiocruz.br

** Centro de Pesquisas em Aquicultura Rodolpho Von Ihering, DNOCS, Pentecoste, Ceará, Brasil.

${ }_{1}$ Pesquisador I-A do CNPq .
} 


\section{Resultados}

Na Tabela 1 são apresentadas as espécies de peixes examinadas e parasitadas pelos diferentes grupos de helmintos e na Figura 1, o gráfico da abundância parasitária.

Do açude Pereira de Miranda 68 (23,4\%) peixes pertencentes a 14 espécies $(77,8 \%)$ encontravam-se parasitados por uma ou mais espécies de helmintos. Das 14 espécies parasitadas, 11 (41 peixes) encontravam-se parasitadas por Monogenea, 4 espécies ( 9 peixes) por Cestoda, uma única espécie (quatro peixes) por Digenea, e uma espécie (dois peixes) pela associação de Monogenea e Cestoda, quatro (dez peixes) por Nematoda e duas espécies (dois peixes) pela associação de Monogenea e Nematoda (Figura 1).

Dos peixes examinados nos viveiros, somente seis não estavam parasitados por helmintos. Dessas 23 espécies, 17 (73,9 $\%)$ encontravam-se parasitadas por helmintos num total de 96 peixes (35,4\%). Das 17 espécies parasitadas, 15 espécies (66 peixes) estavam parasitadas por Monogenea, três espécies por formas imaturas de Digenea, uma espécie (1 peixe) por formas imaturas de Cestoda, uma espécie (1 peixe) pela associação de Monogenea e Digenea, sete (16 peixes) por Nematoda, duas (seis peixes) pela associação de Monogenea e Nematoda.

Tabela 1: Relação de peixes parasitados e examinados no açude Pereira de Miranda e nos viveiros do Centro de Pesquisas em Aquicultura Rodolfo von Ihering. v-ct: variação do comprimento total. v-p: variação do peso. np/ne: no. de peixes parasitados / examinados. M- Monogenea, D- Digenea, C- Cestoda, N- Nematoda

\begin{tabular}{|c|c|c|c|c|c|c|c|c|c|c|c|c|c|c|}
\hline Açude & & & & & & & & Viveiros & & & & & & \\
\hline Hospedeiros & v-ct & v-p & np/ne & 1996 & 1998 & 2000 & 2002 & v-ct & $v-p$ & np/ne & 1996 & 1998 & 2000 & 2002 \\
\hline Anostomidae & & & & & & & & & & & & & & \\
\hline $\begin{array}{l}\text { Leporinus friderici (Bloch, 1794) - } \\
\text { Piau }\end{array}$ & $17-31 \mathrm{~cm}$ & $70-330 \mathrm{~g}$ & $(1 / 2)$ & $x$ & $\mathrm{x}$ & $\mathrm{x}$ & M & $31 \mathrm{~cm}$ & $330 \mathrm{~g}$ & $(0 / 1)$ & $(0 / 1)$ & $x$ & $X$ & $x$ \\
\hline Ariidae & & & & & & & & & & & & & & \\
\hline $\begin{array}{c}\text { Selenaspis herzbergii Bloch, } 1975 \text { - } \\
\text { bagre branco }\end{array}$ & $\mathrm{x}$ & $X$ & $\mathrm{x}$ & $x$ & $\mathrm{x}$ & $\mathrm{x}$ & $x$ & $17,5-65 \mathrm{~cm}$ & $52-3,100 \mathrm{~g}$ & $(1 / 7)$ & $(0 / 1)$ & M & $(0 / 5)$ & $x$ \\
\hline Auchenipteridae & & & & & & & & & & & & & & \\
\hline $\begin{array}{c}\text { Trachychoristes galeatus Linnaeus, } \\
1758 \text { - cangati }\end{array}$ & $11,2-18,5 \mathrm{~cm}$ & $23,5-115 \mathrm{~g}$ & $(2 / 3)$ & $\mathrm{x}$ & $\mathrm{x}$ & $(0 / 1)$ & M & $\mathrm{x}$ & $x$ & $\mathrm{x}$ & $x$ & $\mathrm{X}$ & $\mathrm{X}$ & $\mathrm{x}$ \\
\hline Characidae & & & & & & & & & & & & & & \\
\hline $\begin{array}{c}\text { Astyanax bimaculatus (Linnaeus, } \\
\text { 1758) - piaba }\end{array}$ & $5-11,5 \mathrm{~cm}$ & $2-27 \mathrm{~g}$ & $(11 / 66)$ & $\begin{array}{c}\mathrm{N} \\
\mathrm{M}+\mathrm{M}\end{array}$ & $(0 / 4)$ & $(0 / 15)$ & $(0 / 14)$ & $8-11 \mathrm{~cm}$ & $6-20 \mathrm{~g}$ & $(3 / 30)$ & $x$ & $\begin{array}{l}\mathrm{N}^{*} \\
\mathrm{M}\end{array}$ & (") & $\bar{M}$ \\
\hline $\begin{array}{c}\text { Colossoma brachypomum Cuvier, } \\
1818 \text { - pirapitinga }\end{array}$ & $\mathrm{X}$ & $\mathrm{X}$ & $\mathrm{x}$ & $x$ & X & $x$ & $\mathrm{X}$ & $13-41,5 \mathrm{~cm}$ & $36-1,600 \mathrm{~g}$ & $(10 / 19)$ & M & $\mathrm{M}$ & $M$ & $\begin{array}{l}M \\
D^{*}\end{array}$ \\
\hline $\begin{array}{c}\text { Colossoma macropomum Cuvier, } \\
1818 \text { - tambaqui }\end{array}$ & $X$ & $\mathrm{X}$ & $\mathrm{x}$ & $x$ & $X$ & $x$ & $X$ & $2-35 \mathrm{~cm}$ & $0,5-600 \mathrm{~g}$ & $(6 / 21)$ & M" & $\mathrm{M}$ & $(0 / 3)$ & $(0 / 8)$ \\
\hline $\begin{array}{c}\text { Pellona castelnaeana Valenciennes, } \\
\qquad 1847 \text { - sardinhão }\end{array}$ & $16-18 \mathrm{~cm}$ & $41-51 \mathrm{~g}$ & $(2 / 2)$ & $x$ & $X$ & $x$ & $\mathrm{M}+\mathrm{M}$ & $15-19 \mathrm{~cm}$ & $27-87,8 \mathrm{~g}$ & $(4 / 4)$ & $\mathrm{x}$ & $\mathrm{X}$ & $X$ & $\begin{array}{c}M \\
N \\
N+M\end{array}$ \\
\hline Piaractus mitrei (Berg, 1895) - pacu & X & $\bar{x}$ & $\mathrm{x}$ & $x$ & "' X & $x$ & 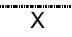 & $30-49 \mathrm{~cm}$ & $470-2,400 \mathrm{~g}$ & $(5 / 5)$ & M" & $\bar{M}$ & 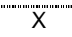 & $X$ \\
\hline $\begin{array}{c}\text { Triportheus angulatus Agassiz, } \\
1889 \text { - sardinha }\end{array}$ & $16,5-19 \mathrm{~cm}$ & $45-65 \mathrm{~g}$ & $(3 / 3)$ & $x$ & "M" & $\mathrm{N}$ & "X' & $13,3-19 \mathrm{~cm}$ & $20-79 \mathrm{~g}$ & $(18 / 22)$ & $\begin{array}{c}N \\
M \\
N+M\end{array}$ & $\bar{M}$ & $\mathrm{M}$ & $\begin{array}{l}\mathrm{N} \\
\mathrm{M}\end{array}$ \\
\hline Cichlidae & & & & & & & & & & & & & & \\
\hline $\begin{array}{c}\text { Astronotus ocellatus (Agassiz, } \\
\text { 1829) - apaiari }\end{array}$ & $18-25 \mathrm{~cm}$ & $134-372 \mathrm{~g}$ & $(2 / 8)$ & $(0 / 3)$ & ' X & M & 'M' & $18-25 \mathrm{~cm}$ & $120-390 \mathrm{~g}$ & $(4 / 12)$ & $\mathrm{N}$ & $0 / 2$ & $0 / 2$ & $\mathrm{M}$ \\
\hline $\begin{array}{c}\text { Cichla ocellaris Bloch \& Schneider, } \\
1801 \text { - tucunaré }\end{array}$ & $9,5-34 \mathrm{~cm}$ & $9-520 \mathrm{~g}$ & $6 / 17$ & $\mathrm{C}+\mathrm{M}$ & $\begin{array}{c}\mathrm{C} \\
\mathrm{M} \\
\mathrm{C}+\mathrm{M}\end{array}$ & $\mathrm{C}$ & "'M" & $9-12,5 \mathrm{~cm}$ & $6-19,5 \mathrm{~g}$ & $(4 / 6)$ & $x$ & $x$ & X' & $\begin{array}{c}M \\
D^{*} \\
M+D^{*}\end{array}$ \\
\hline $\begin{array}{c}\text { Cichlasoma bimaculatum Linnaeus, } \\
1758 \text { - acará }\end{array}$ & $7,8-14 \mathrm{~cm}$ & $10-68 \mathrm{~g}$ & $(1 / 7)$ & $x$ & $\mathrm{X}$ & $\mathrm{C}$ & $(0 / 2)$ & $7,2-7,5 \mathrm{~cm}$ & $7-8 \mathrm{~g}$ & $(0 / 2)$ & $\mathrm{x}$ & $X$ & X' & $(0 / 2)$ \\
\hline $\begin{array}{c}\text { Crenicichla saxatilis (Linnaeus, } \\
\text { 1758) - jacundá }\end{array}$ & $17-23,2 \mathrm{~cm}$ & $58-155,5 \mathrm{~g}$ & $0 / 4$ & $x$ & $\mathrm{X}$ & $(0 / 2)$ & $(0 / 2)$ & $x$ & $x$ & $x$ & $\mathrm{x}$ & $X$ & X & $X$ \\
\hline $\begin{array}{c}\text { Oreochromis hornorumTrewavas, } \\
\text { ?? - tilápia de Zanzibar }\end{array}$ & $\mathrm{X}$ & $\mathrm{X}$ & $\mathrm{x}$ & $x$ & $\mathrm{X}$ & $x$ & $x$ & $21-26 \mathrm{~cm}$ & $155,5-327 \mathrm{~g}$ & $(0 / 4)$ & $x$ & $X$ & $X$ & $(0 / 4)$ \\
\hline $\begin{array}{c}\text { Oreochromis niloticus(Linnaeus, } \\
\text { 1866) -tilápia do Nilo }\end{array}$ & $6-23 \mathrm{~cm}$ & $9-285 \mathrm{~g}$ & $(6 / 21)$ & $(0 / 3)$ & $x$ & $\mathrm{C}^{*}$ & $(0 / 2)$ & $7,5-28 \mathrm{~cm}$ & $8-380 \mathrm{~g}$ & $(11 / 34)$ & $\mathrm{N}$ * & $\mathrm{M}$ & $\mathrm{C}^{*}$ & $M$ \\
\hline Oreochromis sp. - tilápia vermelha & $\mathrm{X}$ & $\mathrm{X}$ & $x$ & $\mathrm{x}$ & $X$ & $x$ & "X' & $14-29 \mathrm{~cm}$ & $15-160 \mathrm{~g}$ & $(6 / 19)$ & $(0 / 3)$ & $\mathrm{M}$ & M & $\mathrm{M}$ \\
\hline $\begin{array}{c}\text { Pseudotrophus zebra????- } \\
\text { zebrinha }\end{array}$ & $\mathrm{X}$ & $\bar{x}$ & $x$ & $x$ & $x$ & $x$ & "X & $0,6-2 \mathrm{~cm}$ & $5-195 \mathrm{~g}$ & $(0 / 14)$ & $(0 / 2)$ & $(0 / 3)$ & "'(0/2) & $(0 / 7)$ \\
\hline $\begin{array}{l}\text { Tilapia rendalli Boulenger, } 1912 \text { - } \\
\text { Tilápia do Congo }\end{array}$ & $17-19 \mathrm{~cm}$ & $310-350 \mathrm{~g}$ & $(0 / 3)$ & $(0 / 3)$ & "'X' & $\mathrm{X}$ & "X & $12-19 \mathrm{~cm}$ & $20-125 \mathrm{~g}$ & $(2 / 13)$ & "N*" & $\mathrm{X}$ & X' & $\mathrm{X}$ \\
\hline Curimatidae & & & & & & & & & & & & & & \\
\hline Curimatus ciliatus????-branquinha & $15-19 \mathrm{~cm}$ & $55-110 \mathrm{~g}$ & $(0 / 4)$ & $\mathrm{x}$ & $\mathrm{X}$ & $(0 / 2)$ & $(0 / 2)$ & $x$ & $\mathrm{x}$ & $\mathrm{x}$ & $\mathrm{X}$ & $\mathrm{X}$ & $x$ & $\mathrm{X}$ \\
\hline $\begin{array}{c}\text { Curimatus elegans Steindachner, } \\
1874 \text { - piabuçu }\end{array}$ & $\mathrm{X}$ & $\mathrm{X}$ & $\mathrm{x}$ & $x$ & $\mathrm{X}$ & $x$ & X & $12 \mathrm{~cm}$ & $35 \mathrm{~g}$ & $(0 / 1)$ & $\mathrm{X}$ & $(0 / 1)$ & X & $\mathrm{X}$ \\
\hline $\begin{array}{c}\text { Prochilodus cearaensis } \\
\text { Steindachner, } 1911 \text { - Curimatã }\end{array}$ & $18-29 \mathrm{~cm}$ & $117-340 \mathrm{~g}$ & $(10 / 13)$ & $\mathrm{M}$ & X & $(0 / 1)$ & $\mathrm{M}$ & $25-34 \mathrm{~cm}$ & $195-650 \mathrm{~g}$ & $(1 / 6)$ & $\mathrm{M}$ & $(0 / 2)$ & "'(0/1) & $\mathrm{X}$ \\
\hline Cyprinidae & & & & & & & & & & & & & & \\
\hline $\begin{array}{l}\text { Cyprinus carpio (Linnaeus, 1958) - } \\
\text { carpa }\end{array}$ & $\mathrm{X}$ & $\mathrm{X}$ & $x$ & $\mathrm{x}$ & $\mathrm{X}$ & $\mathrm{x}$ & & $13,5-33 \mathrm{~cm}$ & $66-480 \mathrm{~g}$ & $(13 / 23)$ & M & M & M & M \\
\hline $\begin{array}{l}\text { Erythrinidae } \\
\end{array}$ & & & & & & & & & & & & & & \\
\hline $\begin{array}{c}\text { Hoplias malabaricusBloch, } 1794 \text { - } \\
\text { traíra }\end{array}$ & $20-40 \mathrm{~cm}$ & $87-1,100 \mathrm{~g}$ & $(8 / 14)$ & $\begin{array}{l}\mathrm{N} \\
\mathrm{M}\end{array}$ & $\begin{array}{l}\mathrm{N} \\
\mathrm{M}\end{array}$ & $(0 / 2)$ & M & $x$ & $x$ & $x$ & $X$ & $x$ & $X$ & $X$ \\
\hline
\end{tabular}


Cont. Tabela 1.

\begin{tabular}{|c|c|c|c|c|c|c|c|c|c|c|c|c|c|c|}
\hline Açude & & \multicolumn{13}{|c|}{ Viveiros } \\
\hline Hospedeiros & v-ct & $v-p$ & $\mathrm{np} / \mathrm{ne}$ & 1996 & 1998 & 2000 & 2002 & $\mathrm{v}-\mathrm{ct}$ & $v-p$ & np/ne & 1996 & 1998 & 2000 & 2002 \\
\hline Loricariidae & & & & & & & & & & & & & & \\
\hline $\begin{array}{c}\text { Loricaria typusBleeker, ??? - cari- } \\
\text { chicote }\end{array}$ & $7,5-23 \mathrm{~cm}$ & $3,5-300 \mathrm{~g}$ & $(4 / 13)$ & M & $\mathrm{M}$ & $(0 / 1)$ & $(0 / 6)$ & $18-22 \mathrm{~cm}$ & $73-130 \mathrm{~g}$ & $(1 / 3)$ & $(0 / 1)$ & M & $(0 / 1)$ & $X$ \\
\hline $\begin{array}{l}\text { Poeciliidae } \\
\end{array}$ & & & & & & & & & & & & & & \\
\hline $\begin{array}{c}\text { Poecilia vivipara Schneider, } 1801 \text { - } \\
\text { guaru }\end{array}$ & $1-6,5 \mathrm{~cm}$ & $1-4 g$ & $(5 / 92)$ & $\mathrm{D}$ & $(0 / 21)$ & $\mathrm{C}$ & $(0 / 18)$ & $2,8-4,5 \mathrm{~cm}$ & $1-2 g$ & $(0 / 12)$ & $X$ & $\mathrm{X}$ & $\mathrm{x}$ & $(0 / 12)$ \\
\hline Sciaenidae & & & & & & & & & & & & & & \\
\hline $\begin{array}{l}\text { Plagioscion squamosissimus } \\
\text { (Heckel, 1840) - pescada }\end{array}$ & $11-32 \mathrm{~cm}$ & $20-330 \mathrm{~g}$ & $(7 / 23)$ & $(0 / 11)$ & $\mathrm{M}$ & M & M & $12-26,8 \mathrm{~cm}$ & $11,5-186 \mathrm{~g}$ & $(6 / 11)$ & $\mathrm{X}$ & M & M & $D^{*}$ \\
\hline Symbranchidae & & & & & & & & & & & & & & \\
\hline $\begin{array}{c}\text { Symbranchus marmoratusBloch, } \\
1795-\mathrm{muçu}\end{array}$ & $45-71 \mathrm{~cm}$ & $105-445 \mathrm{~g}$ & $0 / 2$ & $(0 / 1)$ & $X$ & $(0 / 1)$ & $\mathrm{X}$ & $53-63 \mathrm{~cm}$ & $260-320 \mathrm{~g}$ & $(2 / 2)$ & $\mathrm{X}$ & $\mathrm{N}^{*}$ & $\mathrm{X}$ & $\mathrm{X}$ \\
\hline
\end{tabular}

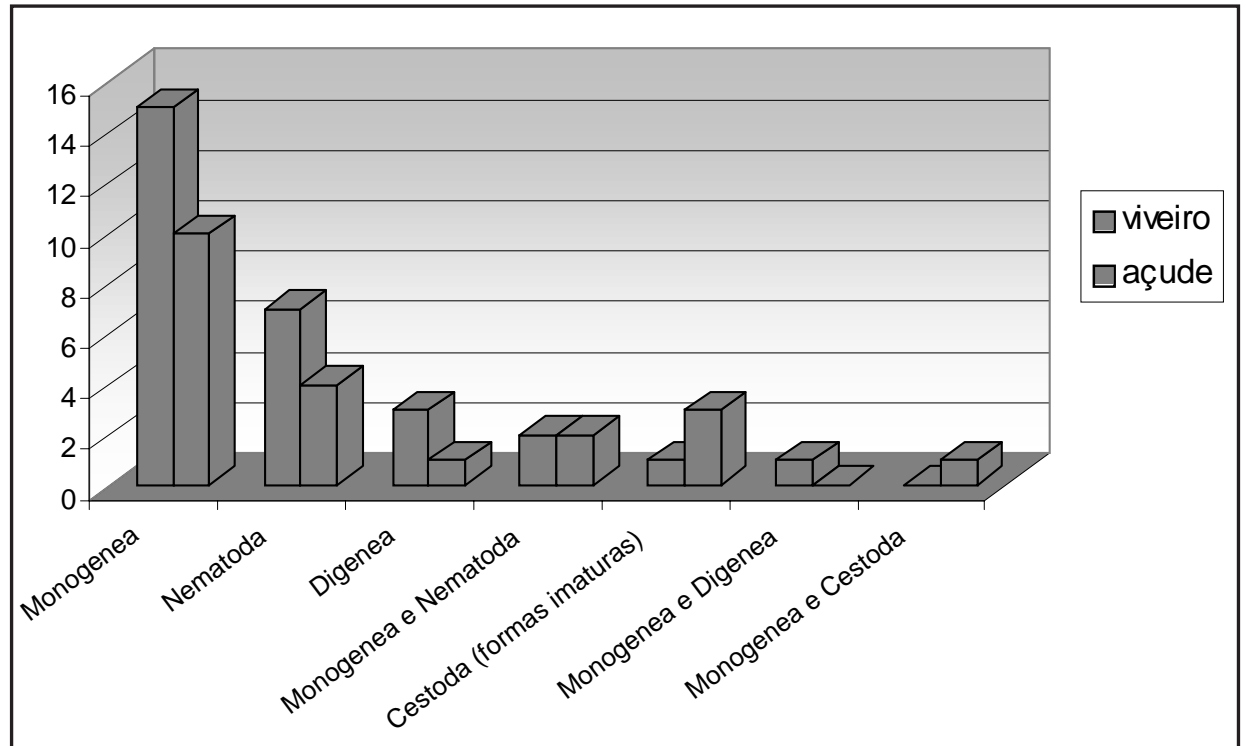

Figura 1: Abundância parasitária dos helmintos nos peixes do DNOCS

\section{Discussão e conclusões}

Nas avaliações parasitológicas realizadas por Békési (1992) nos peixes do DNOCS, foram examinados principalmente oito espécies do C.P. Rodolfo V. Ihering e de outras estações de piscicultura de outros estados. Seus resultados também demonstraram que Monogenea foi o grupo mais representativo entre os helmintos, o que foi confirmado em nosso estudo. A determinação específica dos helmintos encontrados será publicada posteriormente.

O maior índice de parasitismo, representado pelos Monoge-nea, encontrados em 18 das 27 espécies estudadas, tanto nos peixes dos viveiros como nos do açude, pode ser explicado pelo ciclo direto desses parasitos, sem hospedeiros intermediários. Representantes dos Nematoda parasitavam nove espécies de peixes enquanto os Cestoda e os Digenea foram encontrados em quatro espécies. Três espécies de peixes encontravam-se parasitadas pela associação de Monogenea e Nematoda, enquanto somente uma espécie proveniente dos viveiros encontrava-se parasitada pela associação de Monogenea e Digenea (forma imatura) e do açude por Monogenea e Cestoda.

\section{Agradecimentos}

Os autores agradecem à Diretoria do Departamento de Obras contra a Secas (DNOCS) pelas facilidades oferecidas para a realização dos trabalhos.

\section{Referências}

BÉKEZI, L. Evaluation of data on ichtyopathological analyses in the Brazilian Northeast. Ciênc. Cultur, v. 44, p. 400-403, 1992.

BUSH, A. O., LAFFETY, K. D.; LOTZ, J. M.; SHOSTAK, A. W. Parasitology meetsecology on its own terms: Margolis et al. Revisited. J. Parasitol., v. 83, p. 575-583, 1997.

KOHN, A,; FERNANDES, B. M. M.; MACEDO, B.; ABRAMSON, B. Helminths parasites of freshwater fishes from Pirassununga, $\mathrm{P}, \mathrm{Bra}$ sil. Mem. Inst. Oswaldo Cruz, v. 80, p. 327-336, 1985.

KOHN, A.; FERNANDES, B. M. M. Estudo comparativo do helmintos parasitos de peixes do Rio Mogi Guassu, coletados nas excursões realizadas entre 1927 e 1985. Mem. Inst. Oswaldo Cruz, v. 82, p. 483-500, 1987.
KOHN, A.;FERNANDES, B. M. M.;PIPOLO, H. V.; GODOY, M. P. Helmintos Parasitos de Peixes das usinas Hidrelétricas da Eletrosul (Brasil). II Reservatórios de Salto Osório e de Salto Santiago, Bacia do Rio Iguaçu. Mem. Inst. Oswaldo Cruz, v. 83, p. 299-303, 1988.

MACHADO, P. M.; ALMEIDA, S. C.; PAVANELLI, G. C.;TAKEMOTO, R. M. Ecological aspects of endohelminths parasitizing Cichla monoculus Spix, 1831 (Perciformes: Cichlidae) in the Paraná River near Porto Rico, state of Paraná, Brazil. Comp. Parasitol., v. 67, p. 210-217, 2000.

PAVANELLI, G. C., MACHADOM. H.;TAKEMOTO, R. M. Fauna Helmíntica do rio Paraná, região de Porto Rico, Paraná. In: VAZZOLER A. E. A. D. E. M.; AGOSTINHO, A. A.; HAHN, N. S. A planície de inundação do alto rio Paraná, p. 307-329, 1997. 